\title{
Effect of Isotacticity of Linear Poly( $N$-isopropylacrylamide) on its Gelation in Benzyl Alcohol
}

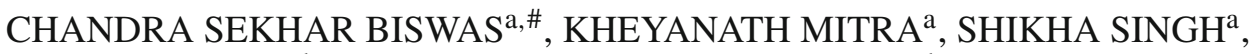 \\ DINESH K PATEL ${ }^{\mathrm{b}}$, BISWAJIT MAITI ${ }^{\mathrm{a}}$, PRALAY MAITI ${ }^{\mathrm{b}}$ and BISWAJIT RAY ${ }^{\mathrm{a}}$ ** \\ ${ }^{a}$ Department of Chemistry, Institute of Science, Banaras Hindu University, Varanasi 221 005, India \\ ${ }^{\mathrm{b}}$ School of Material Science and Technology, Indian Institute of Technology, Banaras Hindu University, \\ Varanasi 221 005, India \\ \#Present address: Nanshan District Key Lab for Biopolymers and Safety Evaluation, College of Materials \\ Science and Engineering and Optoelectronic Engineering Postdoctoral Centre, Shenzhen University, \\ 518060 Shenzhen, China \\ e-mail: biswajitray2003@yahoo.co.in
}

MS received 3 February 2016; accepted 5 April 2016

\begin{abstract}
Thermoreversible gelation of three different isotactic linear poly $(N$-isopropylacrylamide) (PNIPAM)s having meso dyad $(\mathrm{m})$ values 62,68 and $81 \%$ has been observed in benzyl alcohol. All the gels were transparent in nature. SEM image of the dried gels showed fibrillar network morphology. Melting temperature of the gels gradually increased with the increase in the concentration. XRD data of dry polymers and their corresponding dry gels showed shifting in the peak positions. Rheological study showed that stronger gels were formed with increasing isotacticity of PNIPAM while lower isotactic sample exhibited typical polymer melt rheology. The formation of a plunge in the storage modulus as well as in the viscosity plot at the same frequency range indicates the reversible nature of the structure breaking/reformation under frequency sweep. Moreover, the mechanical strength of the gel decreased with increase in temperature. UV-Vis kinetic study also indicated the change in the conformation and aggregation of PNIPAM chains during gelation. Molecular modelling calculation showed that the number of solvent molecules involved in forming gel (polymer-solvent compound) decreased with the increase in the isotacticity of the polymer. Gelation rate of these gels was studied as a function of temperature, concentration and isotacticity using test-tube tilting method. It increased with the increase in the concentration and isoacticity of the polymer, and with the decrease in the temperature. Critical gelation concentration of the gel gradually increased with the decrease in the isotacticity and with the increase in the temperature. All these experimental results indicated that gelation occurs presumably through polymer-solvent compound formation.
\end{abstract}

Keywords. Linear poly $(N$-isopropylacrylamide); isotacticity; thermo reversible gelation; benzyl alcohol.

\section{Introduction}

Thermoreversible, physically cross-linked gels are very important due to different technological applications..$^{1,2}$ Several such gels of different polymers in different solvents were reported in the literature..$^{3-5}$ Out of these, very few reports are about poly( $N$-isopropylacrylamide) (PNIPAM). ${ }^{6-8,10}$ Lin and Cheng reported the thermoreversible gelation of block and star copolymers of poly(ethylene oxide) and PNIPAM of varying architecture in water. ${ }^{6}$ Liu et al., reported the temperature responsive reversible sol-gel transition of PNIPAM- $g$ methylcellulose copolymer hydrogel in water at $25^{\circ} \mathrm{C} .^{7}$ Teodorescu et al., reported the thermogelation properties of PNIPAM- $b$-poly(ethylene glycol)- $b$-PNIPAM

*For correspondence triblock copolymer in water. ${ }^{8}$ Recently, Nandi et al., reported the hybrid hydrogels of riboflavin and PNIPAM via radical polymerization of NIPAM in the presence of $\mathrm{N}, \mathrm{N}$-methylene bisacrylamide cross-linker and varying concentration of riboflavin. ${ }^{9}$ Very recently, Okamoto et al., reported the synthesis of highly isotactic PNIPAM by conventional ${ }^{10}$ and controlled ${ }^{11,12}$ radical polymerization methods. The same group also showed that the phase separation temperature of PNIPAM in water gradually decreased with increase in the isotacticity of the PNIPAM. ${ }^{13}$ Later Hirano et al., reported that the phase separation temperature of PNIPAM in water increases with increase in the syndiotacticity of the PNIPAM. ${ }^{14}$ Tenhu et al., reported the gelation of stereoblock ABA-type PNIPAM in water. ${ }^{15}$ Later, they also reported the spontaneous and thermally induced self-organization of ABA type stereoblock 
PNIPAM in water. ${ }^{16}$ Katsumoto et al., reported the upper critical solution temperature (UCST) type phase separation behaviour of stereocontrolled [meso dyad $\%(m)=44-84)$ PNIPAM in bis(2-methoxyethyl) ether. ${ }^{17}$ Later, Nakano et al., reported the thermoreversible gelation of isotactic rich $(m=64)$ PNIPAM in water at different concentrations below its cloudpoint temperature. ${ }^{18}$ We have reported the synthesis, characterization, and study of the swelling properties of isotactic-rich, chemically cross-linked PNIPAM gel prepared in different methanol-water mixtures in the presence $\mathrm{Y}(\mathrm{OTf})_{3}$ as Lewis acid. ${ }^{19,20}$ Recently, we have reported the synthesis of narrow-dispersed high molecular weight PNIPAM with different isotacticities and observed that the glass transition temperature of these polymers decreased with the increase in their tacticity. ${ }^{21}$ We also studied the effect of isotacticity of PNIPAM on some other physical properties like solubility, thermal decomposition, X-ray diffractometry (XRD), surface tension and viscosity of their solutions in $N$, $N$-dimethylformamide, etc. ${ }^{22}$ In our continuous effort, we are interested to prepare physically cross-linked gel of highly isotactic and high molecular weight PNIPAM in a suitable solvent. In this work, we have explored the thermoreversible gelation of four different isotactic ( $m=47,62,68$ and 81\%) PNIPAMs in benzyl alcohol. Formed gels were characterized by scanning electron microscopy (SEM), differential scanning calorimetry (DSC), XRD, rheological study, UV-Vis spectroscopy, and gelation kinetics study using test-tube tilting method. Quantum chemical calculation was also performed to understand the polymer-solvent interaction in forming the gel. Based on these, a probable mechanism of gelation is suggested.

\section{Experimental}

\subsection{Materials}

Benzyl alchohol (Merck, India) was dried and distilled over ignited calcium oxide. The following different isotactic PNIPAM samples were synthesized according to the literature procedure reported earlier by us. ${ }^{21}$

\subsection{Preparation and Characterization of Gels}

For the preparation of gels, predetermined amount of polymer was dissolved in $0.5 \mathrm{~mL}$ benzyl alcohol at $80^{\circ} \mathrm{C}$ for required time to make a homogeneous solution in the glass vials of $8 \mathrm{~mm}$ internal diameter. For the gelation kinetic study, formed gel in glass vial of $8 \mathrm{~mm}$ internal diameter was heated at $80^{\circ} \mathrm{C}$ to make a homogeneous solution, and then it was quickly transferred to a predetermined temperature isothermal bath $\left( \pm 0.1^{\circ} \mathrm{C}\right)$ and monitored the time until the flow of the solution completely ceased upon tilting. The time required for this is considered as the gelation time. This process was repeated three times for each sample in order to get the average result. The inverse of gelation time is taken as gelation rate. Gelation rate was studied as a function of polymer concentration and temperature. For morphological study by scanning electron microscopy (SEM), gels were dried under vacuum at room temperature for 7 days. The morphology of the above dried gels was recorded directly in a field emission scanning electron microscope (FESEM) apparatus (Philips, Quanta 200F) under vacuum. Differential Scanning Calorimeter (DSC) study was performed with Mettler 832 DSC instrument under $\mathrm{N}_{2}$ atmosphere. The instrument was calibrated with indium before use. The gels were heated from 0 to $80^{\circ} \mathrm{C}$ at the heating rate of $10^{\circ} \mathrm{C} / \mathrm{min}$. The scattering pattern of the dry polymer and gel films was recorded in a X-Ray diffraction apparatus (model Rigaku Miniflex II). The diffractrograms were recorded from $5-50^{\circ}$ angle at the scanning rate $(2 \theta)$ of $2 \%$ min using nickel-filtered $\mathrm{Cu} \mathrm{K} \alpha$ radiation. The frequency dependence oscillatory shear measurement of $15 \%(\mathrm{w} / \mathrm{v}) \mathrm{m}-62,15 \%$ (w/v) $\mathrm{m}-68$ and $6 \%(\mathrm{w} / \mathrm{v})$ gel of m-81 PNIPAM was performed using dynamic frequency sweep test method on Rheologica (model: Nova) using parallel plate geometry $(25 \mathrm{~mm})$ at the desired temperature. The gap between two parallel plates was kept at $1 \mathrm{~mm}$. The data was collected in the frequency range from 0.1 to $100 \mathrm{rad} / \mathrm{sec}$ and with a controlled strain of $1 \%$. During the experiment the strain was kept low to perform the experiment at linear viscoelastic region. The storage moduli and complex viscosities were measured as a function of angular frequency at the specified temperatures. UV-Vis spectra of the polymer solution were taken at 1 minute interval during cooling using Cary 100Bio UV-Vis spectrophotometer (Varian).Quantum chemical calculations of the interactions of different isotactic PNIPAMs (considering degree of polymerization, $x=6$ ) with benzyl alcohol were performed at B3LYP/6-31G** level using Gaussian 03 suits of program. ${ }^{23}$

\section{Results and Discussion}

\subsection{Characteristics of Polymers and Choice of Solvent}

The characteristics of the studied polymers are given in the table 1. It is apparent from the table that all four polymers (m-47, m-62, m-68 and m-81) have molecular 
weights $\left(M_{\mathrm{n}}\right)$ more or less close to 100,000 with polydispersity (PDI) in the range of $1.25-1.34$ and the isotacticity [meso dyad $(m), \%$ ] of these polymers are 47 , 62,68 , and $81 \%$, respectively. We can anticipate that the effect of molecular weight and PDI on the gel properties will be minimum here. The only factor which can predominately affect the gel properties is their isotacticities. It is to be noted that the average tacticity ( $m$ dyad value) of these high molecular weight polymers are reported here. These polymers may have gradient stereostructures, i.e., the $m$ dyad content near the initiating chain-end is higher than that near the terminating chain-end since the $m$ dyad content slightly decreased with an increase in conversion. ${ }^{12}$

We did not observe the gel formation of these PNIPAM samples in different solvents like methanol, ethanol, propanol, $N, N$-dimethyl formamide (DMF), $N, N$-diethyl formamide (DEF), $N, N$-diisopropyl formamide (DIF), $N, N$-dimethyl propionamide (DMP), $N, N$-dimethyl sulfoxide (DMSO), tetrahydrofuran (THF) at room temperature up to $20 \%$ (w/v) concentration. We also did not observe the gel formation of $\mathrm{m}$ 47 PNIPAM sample in benzyl alcohol at room temperature up to $15 \%(\mathrm{w} / \mathrm{v})$ concentration. But, the gel was formed in benzyl alcohol using other (m-62, m-68, and $\mathrm{m}$-81) PNIPAM samples at different temperatures and different concentrations (vide table 2).

Table 1. Characteristics of PNIPAM samples used.

\begin{tabular}{lccc}
\hline Sample & $M_{\mathrm{n}}(\mathrm{g} / \mathrm{mol})$ & $M_{\mathrm{w}} / M_{\mathrm{n}}$ & Tacticity $(\mathrm{m} / \mathrm{r})$ \\
\hline $\mathrm{m}-47$ & 76700 & 1.23 & $47 / 53$ \\
$\mathrm{~m}-62$ & 102000 & 1.25 & $62 / 38$ \\
$\mathrm{~m}-68$ & 93800 & 1.34 & $68 / 32$ \\
$\mathrm{~m}-81$ & 96300 & 1.29 & $81 / 19$ \\
\hline
\end{tabular}

Table 2. Temperature and concentration range studied for different isotactic PNIPAMs.

\begin{tabular}{lccc}
\hline \multicolumn{4}{c}{ Range of Gelation Concentration $(\mathrm{g} / \mathrm{dL})$} \\
\hline Temperature & $\mathrm{m}-62$ & $\mathrm{~m}-68$ & $\mathrm{~m}-81$ \\
\hline $5^{\circ} \mathrm{C}$ & $7-15$ & $6-15$ & $2-9$ \\
$10^{\circ} \mathrm{C}$ & $8-15$ & $7-15$ & $2-9$ \\
$15^{\circ} \mathrm{C}$ & $10-15$ & $8-15$ & $2-9$ \\
$20^{\circ} \mathrm{C}$ & $12-15$ & $10-15$ & $2-9$ \\
$25^{\circ} \mathrm{C}$ & & $11-15$ & $2-9$ \\
$30^{\circ} \mathrm{C}$ & & $13-15$ & $3-9$ \\
$35^{\circ} \mathrm{C}$ & & & $3-9$ \\
$40^{\circ} \mathrm{C}$ & & & $4-9$ \\
$45^{\circ} \mathrm{C}$ & & & $4-9$ \\
$50^{\circ} \mathrm{C}$ & & & $5-9$ \\
$55^{\circ} \mathrm{C}$ & & & $6-9$ \\
\hline
\end{tabular}

\subsection{Formation of Thermoreversible Gel}

Typical photographs of the thermally induced sol-gel transition of $6 \%(\mathrm{w} / \mathrm{v}) \mathrm{m}-81$ PNIPAM sample in benzyl alcohol are shown in figure 1. It clearly shows that transparent gel was formed on cooling from the homogeneous transparent polymer solution formed on heating at $80^{\circ} \mathrm{C}$. The observed sol-gel transition process is reversible. Similar observation was also observed for m-62 and m-68 PNIPAM samples in the studied concentration range of $7-15 \%(w / v)$, and for m-81 PNIPAM sample in the studied concentration range of 2-9\% (w/v). Formation of no gel from m-47 PNIPAM sample up to the concentration of $15 \%(\mathrm{w} / \mathrm{v})$ indicates that such gelation phenomena depends on the tacticity of PNIPAM.

\subsection{Morphological Study by SEM}

SEM micrographs of the dried $15 \%(\mathrm{w} / \mathrm{v})$ gels of m62 and m-68 PNIPAM samples and 9\% (w/v) gel of $\mathrm{m}-81$ PNIPAM are shown in figure 2 (a), (b) and (c), respectively. All images clearly showed the porous matrix with Knoevenagel type morphology in the dried gels. This Knoevenagel network type morphology is a characteristic feature of a thermoreversible polymer gel. $^{24-29}$

\subsection{Differential Scanning Calorimetric Study}

Typical DSC heating thermograms of m-81 PNIPAM gels in benzyl alcohol at three different concentrations $[3,4$, and $5 \%(\mathrm{w} / \mathrm{v})]$ are shown in figure 3 . Endothermic peaks were observed from $3 \%(\mathrm{w} / \mathrm{v})$ concentration onwards. From the DSC thermograms, it is clear

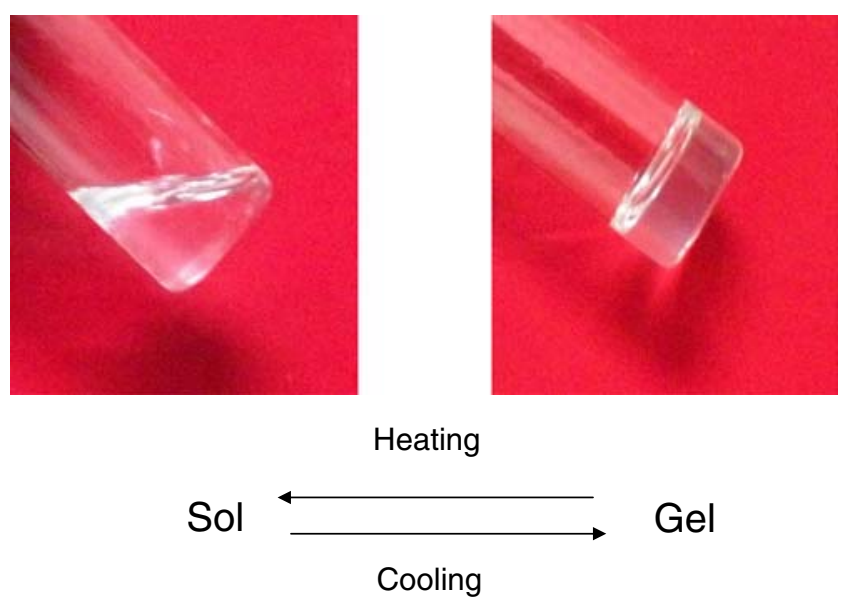

Figure 1. Thermally induced sol-gel transition of $6 \%(\mathrm{w} / \mathrm{v})$ m-81 PNIPAM sample in benzyl alcohol. 

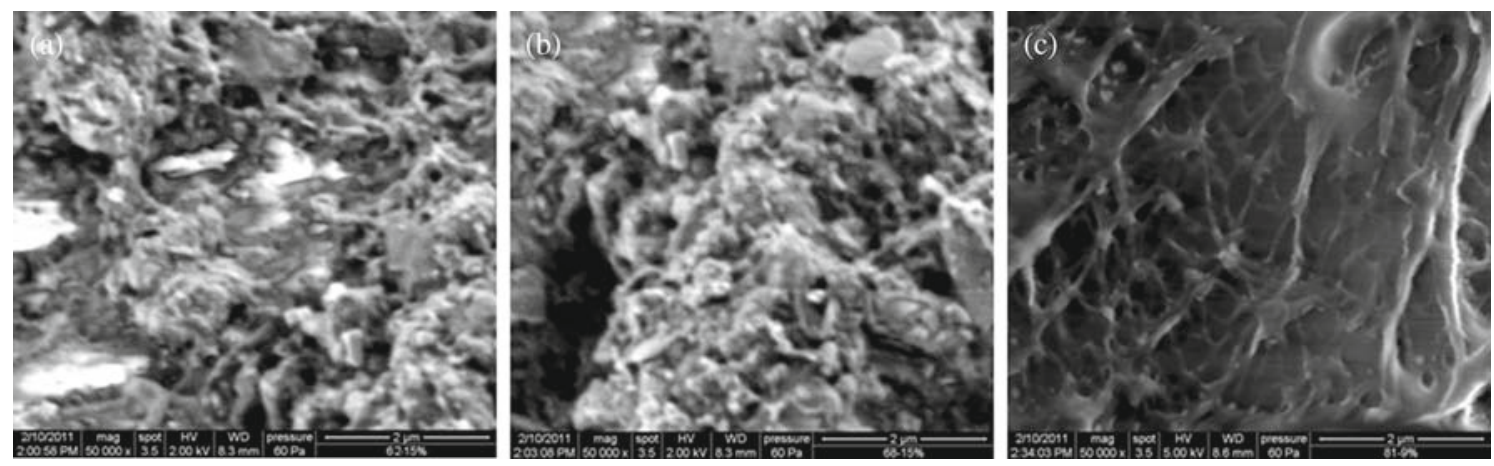

Figure 2. SEM micrographs of the dried (a) $15 \%(w / v)$ gel of m-62 PNIPAM, (b) 15\% (w/v) gel of m-68 PNIPAM and (c) $9 \%(w / v)$ gel of m-81 PNIPAM.

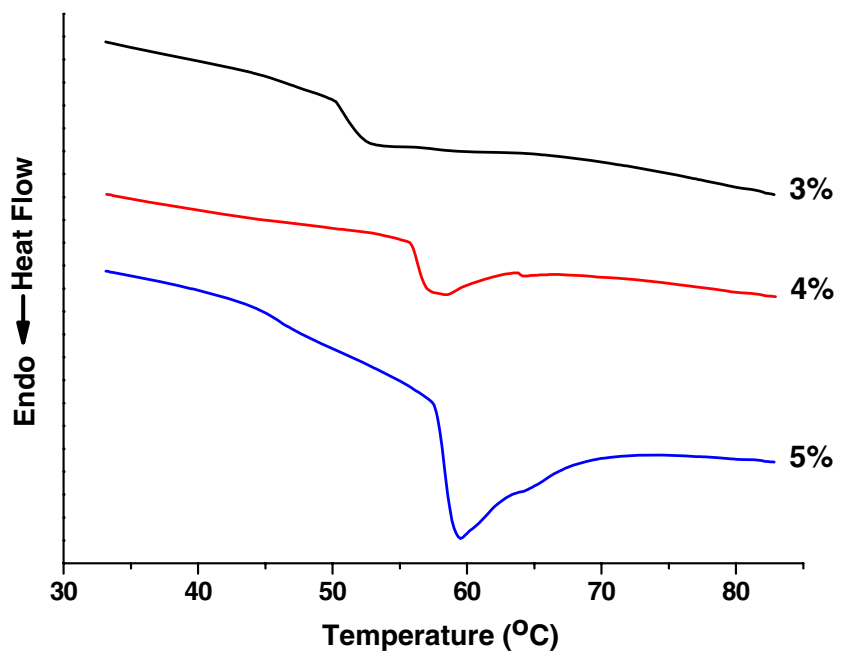

Figure 3. DSC heating thermograms of 3,4 and $5 \%(w / v)$ of m-81 PNIPAM gels in benzyl alcohol.

that the melting point $\left(t_{\mathrm{m}}\right)$ of gels gradually increased with the increase in the concentration of polymer. Here, the gel melting point is a function of concentration. This observation indicates that higher the concentration of the polymer, higher is the density of the physically cross-linked domain and eventually higher is the melting temperature. Similar observations were also reported in the literature. ${ }^{30}$ It is to be noted that no melting point was observed for $7 \%(\mathrm{w} / \mathrm{v}) \mathrm{m}-62$ and m-68 PNIPAM gel samples (figure S1, in Supporting Information).

\subsection{X-Ray Diffraction Study}

Typical XRD patterns of dry m-81 PNIPAM polymer and its dry gels from its 5, 7 and 9\% (w/v) solution in benzyl alcohol are shown in figure 4. The XRD of the polymer has three peaks at $7.33,20.13$ and $40^{\circ}$. The peak obtained at around $7.33^{\circ}$ may be due to the aggregation of the amide bonds of the side chains of

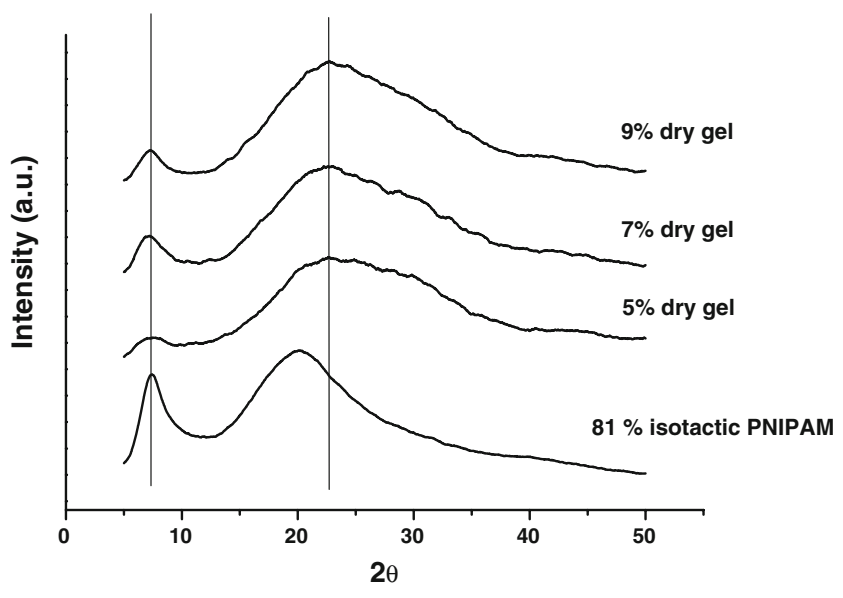

Figure 4. X-ray diffractograms of $\mathrm{m}-81$ dry polymer and its dry gels from 5, 7 and 9\% (w/v) concentrations.

the PNIPAM through hydrogen bonding. The peak at $20.1^{\circ}$ is clearly indicating that the polymer is predominantly amorphous in nature. ${ }^{22}$ This is also supported by the presence of the very weak peak at around $40^{\circ}$. Similar XRD patterns were observed for the dried gels of different concentrations. But, the observed peaks are broader in the polymer gels. The peaks at 20.1 and $40^{\circ}$ of the gels shifted towards higher $2 \theta$ values. The corresponding $d$ spacings $\left(d_{\mathrm{hkl}}\right)$ for both dry polymer and dry gels calculated by using Bragg's equation are presented in table S1 (Supporting Information). The observed higher $d_{\mathrm{hkl}}$ value $(0.441 \mathrm{~nm})$ at $2 \theta=20.1^{\circ}$ of the dry polymer with respect to that $(0.392 \mathrm{~nm})$ of the gels at $2 \theta=22.6^{\circ}$ indicates that some sort of conformational changes occur in the gel structure with respect to the dry polymers although both fall in the amorphous region.

\subsection{Rheological Study}

The rheological study of the m-62, m-68 and m-81 gels were carried out to get the idea about the frequency $(0.1$ to $100 \mathrm{rad} / \mathrm{sec})$ dependant variation of the storage 
modulus, $\mathrm{G}^{/}$and complex viscosity [figures 5(a), (b), and (c)]. The effect of concentration on the rheological properties is presented in figure 5(a) at a particular temperature of $40^{\circ} \mathrm{C} .15 \%$ gel of $\mathrm{m}-62$ shows gradual increase of modulus with increasing frequency following the liquid like behaviour of the material. It is to be mentioned that $15 \%$ gel of m-62 form very weak gel [as evident from the formation of gel at higher concentration (7-15\%) and at lower temperature range (5$20^{\circ} \mathrm{C}$ ). On the other hand, $15 \%$ gel of $\mathrm{m}-68$ is moderately strong as evident from its formation even at higher temperature $\left(5-30^{\circ} \mathrm{C}\right)$. Further, $6 \%$ gel of $\mathrm{m}-81$ is very strong from its formation at very low concentration (2-9\%) and at high temperature $\left(5-55^{\circ} \mathrm{C}\right)$ although its polymer concentration is very low. The storage modulus of $15 \%$ gel of m-68 shows almost constant storage modulus up to the frequency of 1 rad.s ${ }^{-1}$ followed by its increase at higher frequency. On the other hand, $6 \%$ gel of m-81 exhibited constant value of its modulus up to $10 \mathrm{rad} . \mathrm{s}^{-1}$ followed by its increment with increase in frequency. The onset of increasing modulus is considered to be the breakage of network structure and now it is clear that $6 \%$ gel of $\mathrm{m}-81$ contain highest strength as it breaks at 10 rad.s ${ }^{-1}$ of frequency. Similar trend was observed in viscosity measurement (figure S2, in Supporting Information). Slight dip was observed in moderately strong gel (15\% gel of m-68) while considerable dip was noticed in strong gel $(6 \%$ gel of $\mathrm{m}-81)$ where good network structure was already formed. It is worth mentioning that good network structure means relatively bigger physically cross-linked domain. Hence, this study demonstrates the relation between the relative physical cross-linked domain with the strength of gel, which in turn dictates the breakage and reformation of network structure as a function of frequency.

It is to be noted here that for $\mathrm{m}-81$ system, the $\mathrm{G}^{\prime}$ decreases gradually with the increase of temperature in the range of $40-53^{\circ} \mathrm{C}$ over the same angular frequency range [figure 5(b)]. This result indicates that the mechanical strength of the gel decreases with the increase in the temperature. Interestingly, each modulus initially remains same and then shows a plunge within the frequency range of 7-8 $\mathrm{rad} / \mathrm{sec}$ and further increases with the increase in the frequency. The plunge in storage modulus in that range of frequency suggests the reversible nature of the structure breaking/reformation (a)

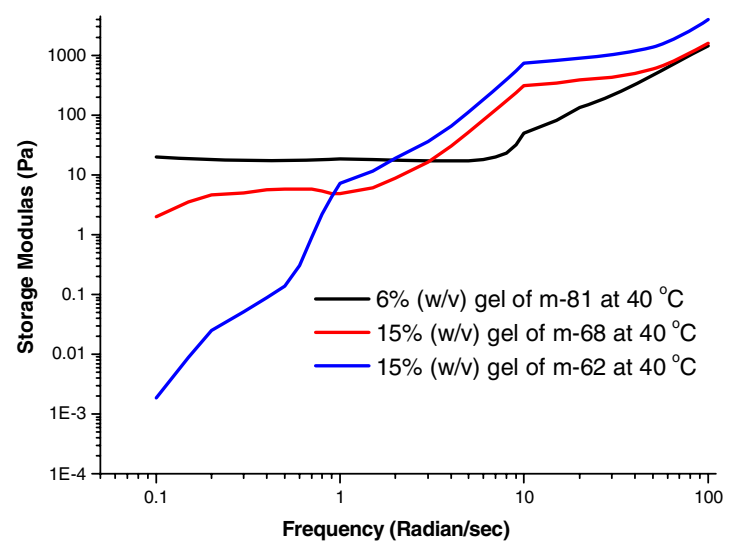

(b)

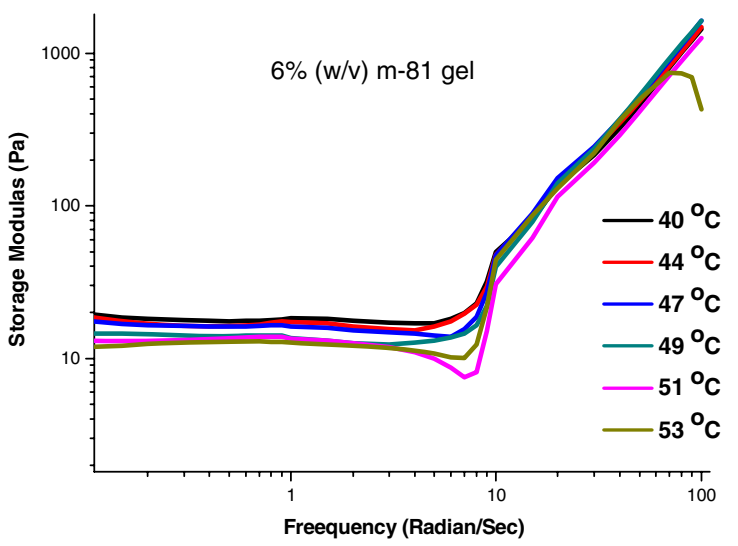

(c)

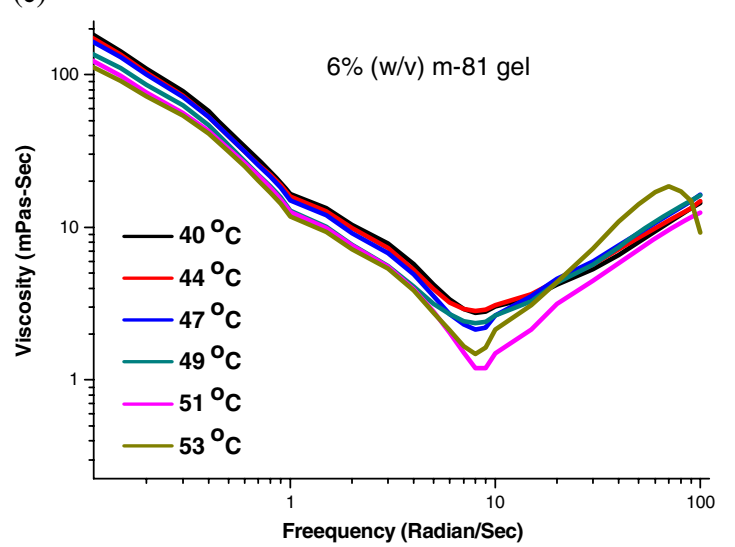

Figure 5. Plots of : (a) storage modulus vs frequency for $15 \%(\mathrm{w} / \mathrm{v}) \mathrm{m}-62,15 \%(\mathrm{w} / \mathrm{v}) \mathrm{m}-68$, and $6 \%$ (w/v) $\mathrm{m}-81$ gels at $40^{\circ} \mathrm{C}$, (b) storage modulus vs frequency for $6 \%(\mathrm{w} / \mathrm{v}) \mathrm{m}-81$ gels at different temperatures, and (c) viscosity vs. frequency for $6 \%(\mathrm{w} / \mathrm{v}) \mathrm{m}-81 \mathrm{gels}$ at different temperatures. 
under frequency sweep. Similar type of observation was also noted for poly(vinylidene fluoride-co-hexafluoro propylene) copolymer systems in phthalates. ${ }^{31}$

The corresponding comparative results of the complex viscosity $v s$. frequency in the temperature range of $40-53^{\circ} \mathrm{C}$ are shown in the figure 5 (c). In general, viscosity decreases with increase in temperature throughout the measured frequency range. Moreover, at a constant temperature, viscosity decreases initially with increasing frequency followed by gradually increasing tendency. This trend suggests the existence of a lowest viscosity frequency where network structure of the gels break. Subsequent increase of viscosities at higher frequency is due to reformation of network structure at higher frequency. Hence, we can conclude that the rheological properties of these gels vary with temperature as well as frequency. Lower isotacticity sample shows very weak gel with improper network structure while the strength of network gradually increases with isotacticity indicating typical rheological pattern of gel at high isotacticity sample (m-81).

\section{$3.7 \quad$ UV-Vis Study}

In order to understand the gelation process, a typical time dependent UV-Vis spectroscopic study of $\mathrm{m}-81$ PNIPAM in benzyl alcohol [2\% (w/v)] solution was performed and the corresponding results are shown in figure S3 (a) and (b) (Supporting Information). The peak at $313 \mathrm{~nm}$ is due to the $\Pi-\Pi^{*}$ transition of the amide carbonyl group of PNIPAM, and this peak shifts from 313 to lower wavelength region with time. Though no definite reason for this blue shift is known, it may be

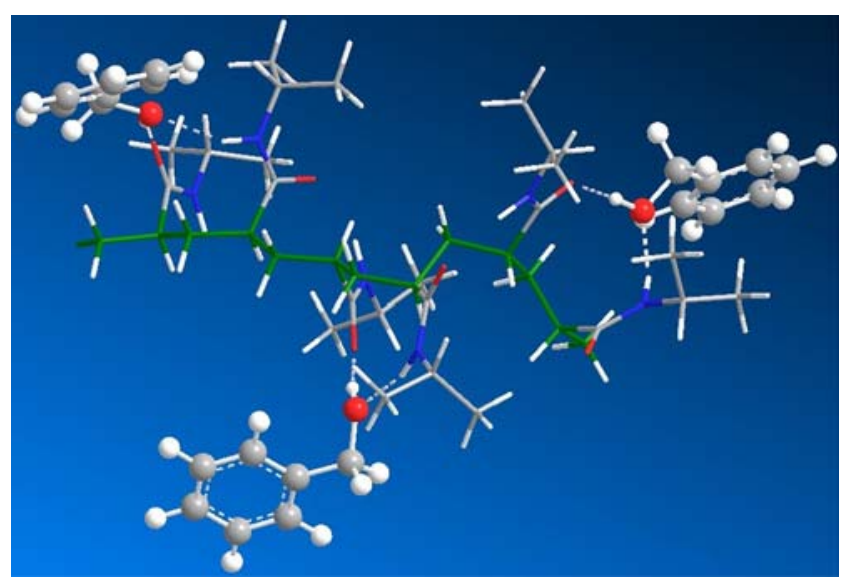

Figure 6. Interaction of $m=66 \%$ isotactic PNIPAM having degree of polymerization, $\mathrm{x}=6$ with benzyl alcohol. [Colors and atoms: white for $\mathrm{H}$ atom, grey for $\mathrm{C}$ atom (except backbone carbon atoms which are shown in green), blue for $\mathrm{N}$ atom and red for $\mathrm{O}$ atom]. attributed to the changes in the PNIPAM-benzyl alcohol interactions during gel formation with gradual decrease in the temperature from $80^{\circ} \mathrm{C}$ to room temperature. Apart from the blue shift, the absorbance value gradually increases with increase in time and then levelled off around 8 min [figure S3(b), in Supporting Information]. The increase in the absorbance value with time indicates that the formation of the PNIPAM-solvent compound takes place during gelation process.

\subsection{Quantum Chemical Calculations}

In order to get the idea about the interaction of benzyl alcohol with different isotactic PNIPAM, we have studied the quantum chemical calculation of interaction of $m=66 \%$ and $83 \%$ isotactic PNIPAMs [having degree of polymerization, $x=6$ ] with benzyl alcohol. Figures 6 and 7 show the corresponding results for $66 \%$ and $83 \%$ isotactic PNIPAM systems, respectively. It shows that three benzyl alcohol molecules can form complex with $m=66 \%$ isotactic PNIPAM (figure 6), whereas two benzyl alcohol molecules can form complex with $m=83 \%$ isotactic PNIPAM (figure 7). So, it is apparent that, with the increase in the isotacticity of the PNIPAM, the interaction between PNIPAM and benzyl alcohol gradually decreases at the cost of the increase in the intramolecular interaction through H-bonding and the van der Waals (dispersion) forces. Consequently, the solubility of the polymer gradually decreases with increase in the isotacticity. Thus, quantum mechanical calculation supports the results observed in the previous sections.

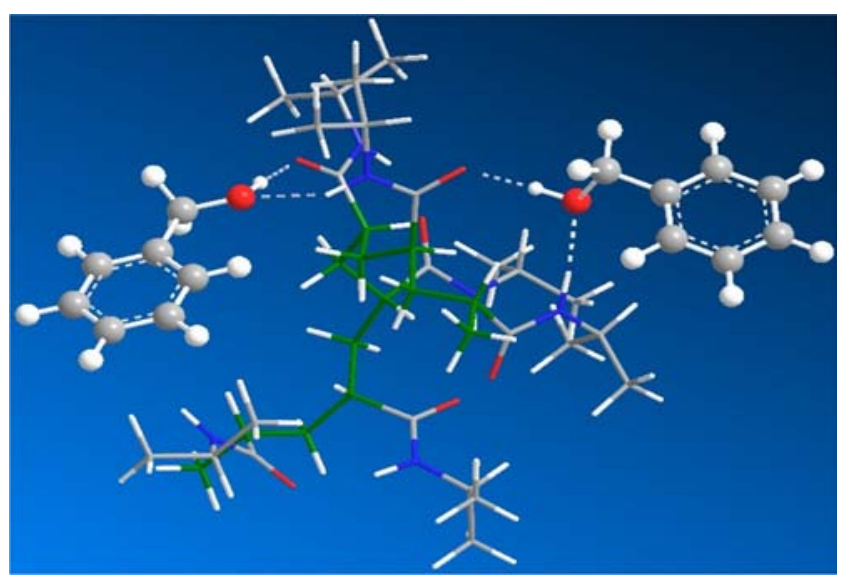

Figure 7. Interaction of $m=83 \%$ isotactic PNIPAM having degree of polymerization, $\mathrm{x}=6$ with benzyl alcohol. [Colors and atoms: white for $\mathrm{H}$ atom, grey for $\mathrm{C}$ atom (except backbone carbon atoms which are shown in green), blue for $\mathrm{N}$ atom and red for $\mathrm{O}$ atom]. 


\subsection{Gelation Rate}

The gelation rate is generally expressed as the inverse of the gelation time. Here, the gelation time is considered as the time required to cease the flow of the polymer solution using test tube tilting method. The temperature and concentration ranges studied for different PNIPAM samples are shown in table 2. m-62 PNIPAM sample was studied in the temperature range $5-20^{\circ} \mathrm{C}$ and the concentration range $7-15 \%(w / v)$. m-68 PNIPAM sample was studied in the temperature range $5-30^{\circ} \mathrm{C}$ and the concentration range 6-15\% (w/v); and m-81 PNIPAM sample was studied in the temperature range $5-55^{\circ} \mathrm{C}$ and the concentration range $2-9 \%(\mathrm{w} / \mathrm{v})$. The gelation rates of $\mathrm{m}-62, \mathrm{~m}-68$, and $\mathrm{m}-81$ PNIPAM gels are plotted against concentration at the specified temperatures and also against temperature at the specified concentration, which are shown in figures S4, S5, and S6 (in Supporting Information).

It is clear from these figures that, in all cases, gelation rate increases exponentially with the increase in polymer concentration at a specific temperature and also linearly with the decrease in temperature at a specific polymer concentration, as expected. ${ }^{24-29,32}$ Moreover, the corresponding plots of the gelation rate $v s$. the isotacticity of the corresponding PNIPAMs at $5^{\circ} \mathrm{C}$ and $10^{\circ} \mathrm{C}$ are shown in figures 8 (a) and (b), respectively. It is clear from this figure that gelation rate initially increases little on increasing the tacticity of PNIPAM from $m=62 \%$ (m-62) to $m=68 \%(\mathrm{~m}-68)$ and then increases considerably with the increase in the isotacticity to $m=81 \%$ (m-81). This is presumably due to the variation in the interaction of benzyl alcohol with the PNIPAMs of different isotacticities. When the isotacticity of PNIPAM is low ( $m=62 \%$ ), the solubility of the PNIPAM in benzyl alcohol is possibly higher due to the stronger interaction between solvent and polymer

(a)

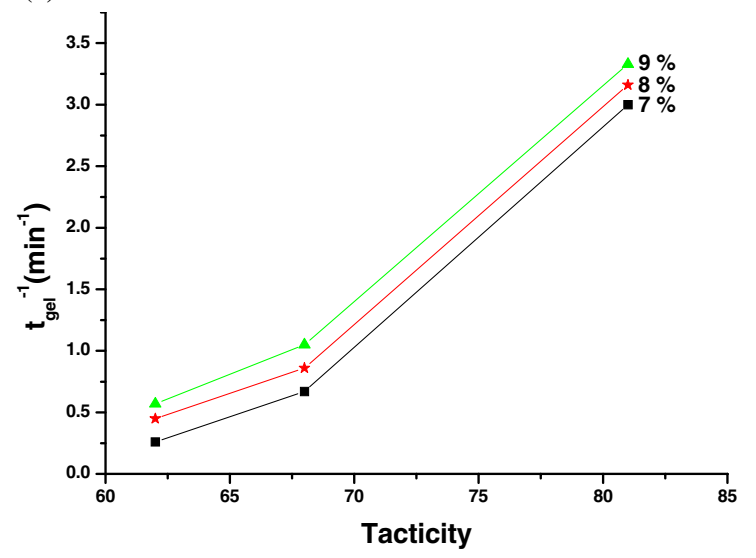

(preferably through hydrogen bonding between amide group of PNIPAM and -OH group of benzyl alcohol) owing to the random distribution of the amide group in both the sides of main polymer chain. Due to such higher solubility, the time required for the gelation is longer. On increasing the isotacticity of PNIPAM from $m=62 \%$ to $m=68 \%$, the interaction between polymer and solvent possibly decreases at the cost of the formation of stronger intra-chain interactions among the side chain groups [H-bonding among amide groups and van der Waals (dispersion) forces among isopropyl groups] $]^{21}$ and eventually gelation occurs within a relatively short period. Upon further increase in the isotacticity to $m=81 \%$, the interaction of PNIPAM with benzyl alcohol possibly decreases drastically and accordingly, the rate of gelation also increases considerably. Quantum chemical calculation of the interaction of benzyl alcohol with the PNIPAM of different isotacticities discussed in section 3.8 supports this explanation. Therefore, the gelation rate of PNIPAM also depends on the tacticity of the polymer apart from the temperature and the polymer concentration.

The extrapolation of each gelation rate $v s$. concentration curve at a specific temperature (T) [figures $\mathrm{S} 4(\mathrm{a})$, $\mathrm{S} 5(\mathrm{a})$ and $\mathrm{S6}$ (a) in $\mathrm{SI}$ ] to the concentration (X-) axis yields the corresponding critical gelation concentration $\left(C^{*}\right),{ }^{25,26}$ which is the minimum concentration required to form a gel at a particular temperature. It is apparent from these figures that $C^{*}$ increases with the increase in the gelation temperature in all PNIPAM samples. ${ }^{25,26}$ The corresponding plots, (i) $\mathrm{C}^{*} v s$. gelation temperature of different isotactic PNIPAM gels, and (ii) $\mathrm{C}^{*}$ $v s$. isotacticity $(m \%)$ of PNIPAMs at different gelation temperatures are shown in figures 9 (a) and (b), respectively. It shows clearly that, at a particular temperature, $\mathrm{C}^{*}$ depends on the tacticity of the polymer. For example, at $10^{\circ} \mathrm{C}$, the $\mathrm{C}^{*}$ of $\mathrm{m}-62, \mathrm{~m}-68$, and $\mathrm{m}-81$ are

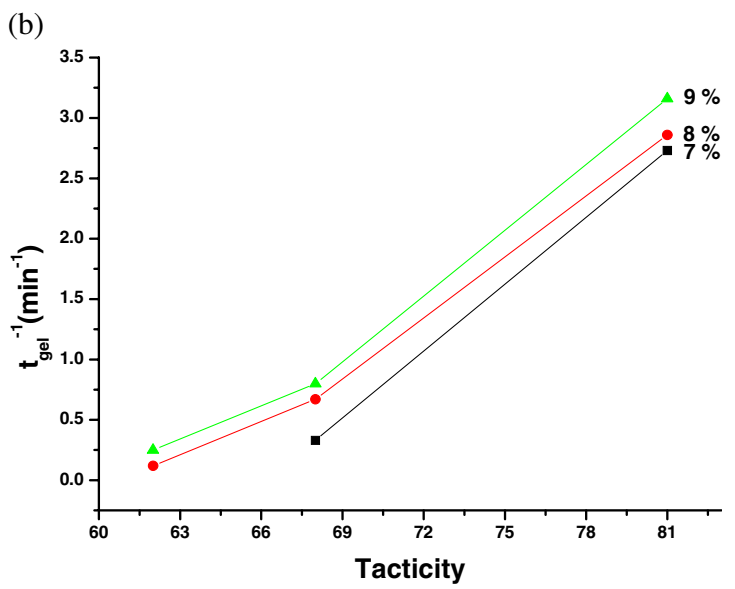

Figure 8. Variation of gelation rate with the tacticity $(m \%)$ of PNIPAM at (a) $5^{\circ} \mathrm{C}$ and (b) $10^{\circ} \mathrm{C}$. 
7.55, 6.02, and $1.16 \mathrm{~g} / \mathrm{dL}$, respectively. Moreover, this increase in $\mathrm{C}^{*}$ with temperature is steeper with the PNIPAM having lower isotacticity [figure 9 (a)]. In addition, at a particular temperature, $C^{*}$ decreases with the increase in the isotacticity [figure 9 (b)] and the rate of decrement of $\mathrm{C}^{*}$ is considerable when the isotacticity of PNIPAM increases from $m=68 \%$ to $m=81 \%$. Such a tacticity dependency of $\mathrm{C}^{*}$ is also possibly due to the gradual decrease in the interaction of benzyl alcohol with the higher isotactic PNIPAMs as discussed above on gelation rate.

In order to get the idea about the enthalpy of gelation for different isotactic PNIPAMs, we have used Eldridge and Ferry ${ }^{30}$ relationship between the polymer concentration (C) and the gelation (or melting) temperature (T):

$$
\log \mathrm{C}=\left(\Delta \mathrm{H}^{\mathrm{o}} / 2.303 \mathrm{RT}\right)+\text { Constant }
$$

where, $\Delta \mathrm{H}^{\circ}=$ heat of reaction to produce $1 \mathrm{~mol}$ of cross-links, $\mathrm{C}=$ polymer concentration $(\mathrm{mole} / \mathrm{L}), \mathrm{T}=$ gelation (or, melting) temperature $(\mathrm{K})$. For different isotactic PNIPAM systems, logarithmic values of $\mathrm{C}^{*} \mathrm{~s}$ at different temperatures are plotted against the inverse of the corresponding temperature (1/T) (figure 10). The figure shows that the data, for each PNIPAM system within the studied temperature range, fit well in a straight line. The calculated $\Delta \mathrm{H}^{\circ}$ values for $\mathrm{m}-62, \mathrm{~m}-68$ and $\mathrm{m}-81$ PNIPAM systems from the corresponding least square slopes are $-16.9,-17.0$, and $-21.1 \mathrm{~kJ}$ $\mathrm{mol}^{-1}$, respectively. So, $\Delta \mathrm{H}^{\circ}$ value is not changed significantly with the increase of the isotacticity of PNIPAM from $m=62$ to $68 \%$, but, on further increase of the isotacticity to $m=81 \%$, there is a considerable increase in $\Delta \mathrm{H}^{\circ}$ value. Therefore, the gelation process is more favourable with higher isotactic PNIPAM. It is possibly due to the relatively easier formation of physical cross-linking junction through polymer-solvent complexation.

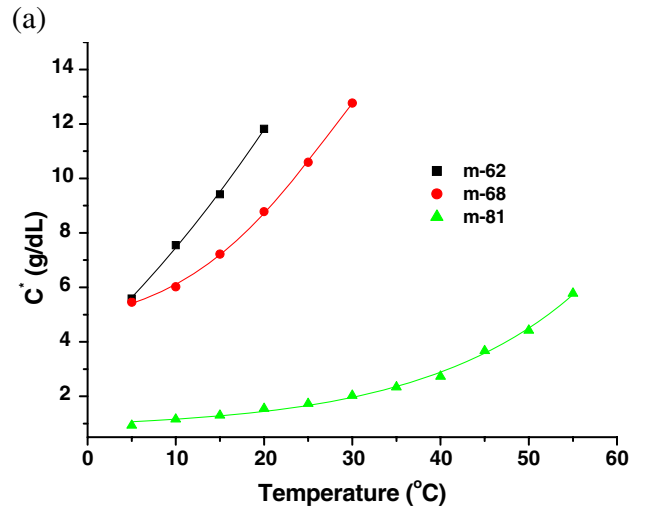

In order to get the idea about the gelation process, we have also plotted the double logarithmic plots of the $t_{\text {gel }}^{-1}$ $v s$. the reduced overlap concentration $\left[\left(\mathrm{C}-\mathrm{C}^{*}\right) / \mathrm{C}^{*}\right]$ at a particular temperature ${ }^{25}$ for $\mathrm{m}-62, \mathrm{~m}-68$ and $\mathrm{m}-81$ PNIPAM systems [figure S7 (a), (b), and (c), respectively, in Supporting Information]. For all PNIPAM systems, it is showing a linear relationship between $\log \left(t_{\text {gel }}^{-1}\right)$ vs. $\log \left[\left(\mathrm{C}-\mathrm{C}^{*}\right) / \mathrm{C}^{*}\right]$ with a positive slope $(n)$. The corresponding calculated $n$ values from the least-squares slopes of the plots are presented in table S2 (a), (b), and (c) (Supporting Information), respectively. The average $n$ values for m-62, m-68, and m-81 PNIPAM systems are $0.71,0.64$, and 0.65 , respectively. These results indicate that the present gelation process occurs above a critical polymer concentration at a particular temperature for a specific isotactic polymer and the gelation rate increases with the increase in reduced overlap concentration $\left[\left(\mathrm{C}-\mathrm{C}^{*}\right) / \mathrm{C}^{*}\right]$ with power $n$. Therefore,

$$
\mathrm{t}_{\mathrm{gel}}^{-1} \propto f(\mathrm{C}) \propto\left[\left(\mathrm{C}-\mathrm{C}^{*}\right) / \mathrm{C}^{*}\right]^{\mathrm{n}}
$$

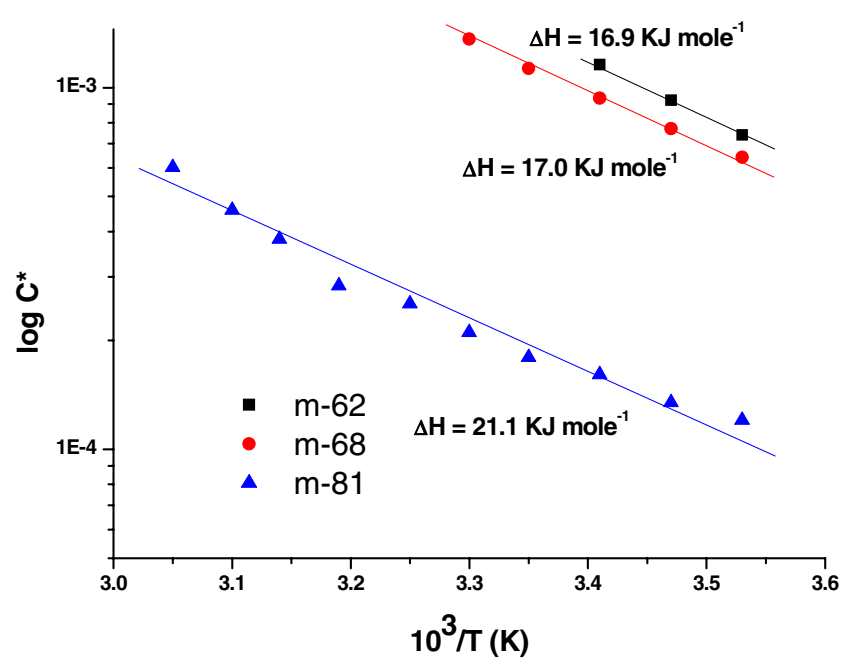

Figure 10. Comparison of change in $\Delta \mathrm{H}^{\circ}$ for the gelation of different isotactic PNIPAMs.

(b)

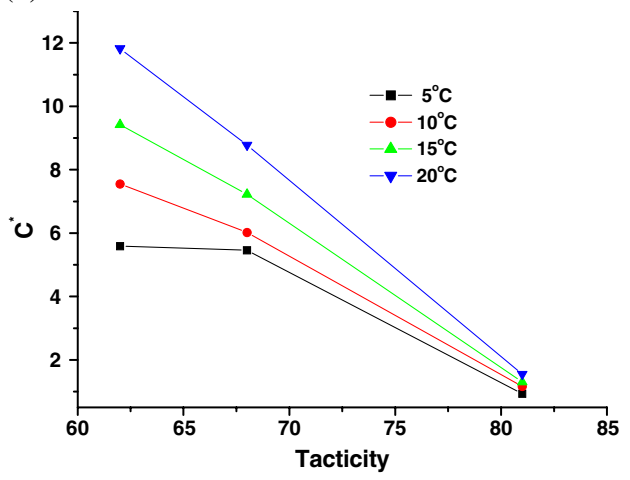

Figure 9. Variation of $\mathrm{C}^{*}$ with (a) temperature and (b) tacticity $(m \%)$ of different isotactic PNIPAMs. 
According to the Percolation theory for threedimensional lattice, $n$ should have the value of $0.45 .^{33}$ Moreover, according to the Flory-Stockmayer theory for Bethe lattice, $n$ should have the value equal to unity. ${ }^{34}$ In our cases, the values come in between these two. Therefore, PNIPAM gel in benzyl alcohol contains intermediate nature of these two lattices.

\subsection{Possible Mechanism of Gelation}

From the above experimental results for the gelation of different isotactic PNIPAMs in benzyl alcohol, a probable gelation mechanism involving formation of polymer-solvent compound via hydrogen bonding (figures 6 and 7) as well as hydrophobic interaction can be assumed. Such a mechanism was first proposed by Guenet et al., to explain the thermoreversible gelation of isotactic polystyrene. ${ }^{35,36}$ At high temperature, chain flexibility and mobility of solvated polymer as well as the mobility of solvent will be high enough due to higher kinetic energy and eventually the formation of polymer-solvent compound will be unfavorable. As temperature goes down, chain flexibility and mobility of the solvated polymer and solvent shall be less, and consequently, polymer-solvent compound formation will be favorable. For a specific isotactic polymer, at a particular temperature, polymer-solvent compound formation will be more favorable with the increase of polymer concentration due to the formation of physically cross-linked domain in higher concentration and eventually gel will be formed above a critical gelation concentration. Moreover, at a particular temperature and concentration of polymer, chain flexibility of solvated polymer will gradually decrease with the increase in the polymer isotacticity ${ }^{21}$ and as a result, the polymer-solvent compound formation will be more favorable with higher isotactic polymer. In order to understand clearly the mechanism of gelation of these PNIPAMs in benzyl alcohol, other studies like UV-Vis, ${ }^{1} \mathrm{H}$ NMR, rheological, small angle $\mathrm{X}$-ray scattering and dynamic light scattering studies are in progress.

\section{Conclusions}

Thermoreversible gelations of three different isotactic PNIPAMs having meso dyad $(m)$ values 62,68 and $81 \%$ have been observed in benzyl alcohol. All the gels are transparent in nature. SEM image of the dried gels shows Knoevenagel network morphology. Moreover, the gel melting temperature of the gels gradually increased with increase in concentration. XRD data of dry polymer and their corresponding dry gels showed shifting in the peak positions. Rheological properties of these gels varied with temperature as well as frequency. Lower isotacticity sample showed very weak gel with improper network structure while the strength of network gradually increased with isotacticity indicating typical rheological pattern of gel at high isotacticity sample (m-81). UV-Vis kinetic study also indicated the change in the conformation and aggregation of PNIPAM chains during gelation. Molecular modelling calculation showed that the number of solvent molecules involved in forming gel (polymer-solvent complex) decreased with increase in isotacticity of the polymer. Gelation rate of these gels has been studied as a function of temperature, concentration and isotacticity using test-tube tilting method. It increased with increase in concentration and isoacticity, and with decrease in temperature. Critical gelation concentration of the gel gradually increased with decrease in the isotacticity and with increase in the temperature. All these experimental results indicated that gelation occurs presumably through polymer-solvent compound formation.

\section{Supplementary Information (SI)}

Results of X-ray analysis data of m-81 PNIPAM and its dry gels, DSC thermograms of m-62 and m-68 PNIPAM gels, plots of complex viscosity $v s$. frequency of gels, UV-Vis spectral changes during gelation, gelation kinetics, and plot of $\log t_{\text {gel }}^{-1}$ vs. $\log \left[\left(\mathrm{C}^{-} \mathrm{C}^{*}\right) / \mathrm{C}^{*}\right]$ are shown in Supplementary Information which is available at www.ias.ac.in/chemsci.

\section{Acknowledgements}

BR gratefully acknowledges the financial support from the Council of Science and Industrial Research, Government of India, through Grant no. 02(0002)/11/EMR-II.

\section{References}

1. Reinecke H, Mijangos C, Brulet A and Guenet J M 1997 Macromolecules 30959

2. Poux S, Malik S, Thierry A, Dosiere M and Guenet J M 2006 Polymer 475596

3. Clark A H and Ross-Murphy S B 1987 Adv. Polym. Sci. 8357

4. Te Nijenhuis K 1997 Adv. Polym. Sci. 1301

5. Guenet J M (Ed.) 1992 In Thermoreversible gelation of polymers and biopolymers (London: Academic Press)

6. Lin H H and Cheng Y L 2001 Macromolecules 343710

7. Liu W, Zhang B, Lu W W, Li X, Zhu D, Yao K D, Wang Q, Zhao C and Wang C 2004 Biomaterials 253005

8. Teodorescu M, Negru I, Stanescu P O, Draghici C, Lungu A and Sarbu A 2010 React. Funct. Polym. 70790 
9. Chakraborty P, Bairi P, Roy B and Nandi A K 2014 RSC Adv. 454684

10. Ishobe Y, Fujioka D, Habaue S and Okamoto Y $2001 \mathrm{~J}$. Am. Chem. Soc. 297180

11. Ray B, Isobe $\mathrm{Y}$, Morioka K, Habaue S, Okamoto Y, Kamigaito M and Sawamoto M 2003 Macromolecules 36543

12. Ray B, Isobe $\mathrm{Y}$, Habaue S, Matsumoto K, Okamoto Y, Kamigaito M and Sawamoto M 2004 Macromolecules 371702

13. Ray B, Okamoto Y, Kamigaito M, Sawamoto M, Seno K-I, Kanaoka S and Aoshima S 2005 Polym. J. 37234

14. Hirano $T$, Okumura $Y$, Kitajima $H$, Seno $M$ and Sato T 2006 J. Polym. Sci. Part A: Polym. Chem. 444450

15. Hietala S, Nuopponen M, Kalliomaki K and Tenhu $\mathrm{H}$ 2008 Macromolecules 412627

16. Nuopponen M, Kalliomaki K, Aseyev V and Tenhu H 2008 Macromolecules 414881

17. Koyama M, Hirano T, Ohno K and Katsumoto K 2008 J. Phys. Chem. B 11210854

18. Nakano S, Ogiso T, Kita R, Shinyashiki N, Yagihara S, Yoneyama M and Katsumoto Y 2011 J. Chem. Phys. 135 114903

19. Biswas C S, Patel V K, Viswakarma N K, Mishra A K, Saha S and Ray B 2010 Langmuir 266675

20. Biswas C S, Viswakarma N K, Patel V K, Mishra A K, Saha S and Ray B 2012 Langmuir 287014

21. Biswas C S, Patel V K, Vishwakarma N K, Tiwari V K, Maiti B, Maiti P, Kamigaito M, Okamoto Y and Ray B 2011 Macromolecules 445822
22. Biswas C S, Mitra K, Singh S, Ramesh K, Misra N, Maiti B, Panda A K, Maiti P, Kamigaito M, Okamoto Y and Ray B 2016 Colloid Polym. Sci. 294399

23. Frisch M J et al. 2007 Gaussian 03, Revision E-01 (Gaussian Inc.: Wallingford CT)

24. Sannigrahi A, Arunbabu D and Jana T 2006 Macromol. Rapid Commun. 271962

25. Mal S, Maiti P and Nandi A K 1995 Macromolecules 282371

26. Mutin P H, Guenet J M, Hirsch E and Candau S J 1988 Polymer 2930

27. Dikshit A K and Nandi A K 2000 Macromolecules 332616

28. Dasgupta D, Malik S, Thierry A, Guenet J M and Nandi A K 2006 Macromolecules 396110

29. Malik S, Jana T and Nandi A K 2001 Macromolecules 34275

30. Eldridge J E and Ferry J D 1954 J. Phys. Chem. 58992

31. Yadav J P, Aswal V K, Sastry P U, Patra A K and Maiti P 2009 J. Phys. Chem. B 11313516

32. Sannigrahi A, Ghosh S, Maity S and Jana T 2011 Polymer 524319

33. Stauffer D and Coniglio M A 1982 Adv. Polym. Sci. 44 103

34. Zallen R (Ed.) 1983 In The Physics of Amorphous Solid (New York: John Wiley) p.135

35. Guenet J M 1987 Macromolecules 202874

36. Klein M, Brulet A, Boue F and Guenet J M 1991 Polymer 321943 